\title{
Critical role of CdSe nanoplatelets in color-converting CdSe/ZnS nanocrystals for InGaN/GaN light-emitting diodes
}

\author{
Namig Hasanov, ${ }^{1}$ Vijay Kumar Sharma, ${ }^{1}$ Pedro Ludwig Hernandez Martinez, ${ }^{1}$ \\ Swee Tiam Tan, ${ }^{1}$ and Hilmi Volkan Demir ${ }^{1,2,3, *}$ \\ ${ }^{1}$ LUMINOUS! Centre of Excellence for Semiconductor Lighting and Displays, School of Electrical and Electronic Engineering, Nanyang \\ Technological University, 50 Nanyang Avenue, Singapore 639798, Singapore \\ ${ }^{2}$ School of Physical and Mathematical Sciences, Nanyang Technological University, Singapore, 21 Nanyang Link, Singapore 639798, Singapore \\ ${ }^{3}$ Department of Electrical and Electronics, Department of Physics, and UNAM-Institute of Material Science and Nanotechnology, Bilkent \\ University, TR-06800, Ankara, Turkey \\ *Corresponding author: volkan@stanfordalumni.org
}

Received 7 April 2016; revised 9 May 2016; accepted 22 May 2016; posted 23 May 2016 (Doc. ID 262542); published 15 June 2016

\begin{abstract}
Here we report CdSe nanoplatelets that are incorporated into color-converting CdSe/ZnS nanocrystals for InGaN/ $\mathrm{GaN}$ light-emitting diodes. The critical role of $\mathrm{CdSe}$ nanoplatelets as an exciton donor for the color conversion was experimentally investigated. The power conversion efficiency of the hybrid light-emitting diode was found to increase by $23 \%$ with the incorporation of the CdSe nanoplatelets. The performance enhancement is ascribed to efficient exciton transfer from the donor CdSe nanoplatelet quantum wells to the acceptor $\mathrm{CdSe} / \mathrm{ZnS}$ nanocrystal quantum dots through Förster-type nonradiative resonance energy transfer. (๑) 2016 Optical Society of America
\end{abstract}

OCIS codes: (160.4236) Nanomaterials; (230.3670) Light-emitting diodes; (260.2160) Energy transfer.

http://dx.doi.org/10.1364/OL.41.002883

Colloidal semiconductor nanocrystals are promising optical components thanks to their high quantum efficiency, low-cost synthesis, narrow luminescence, long lifetime, and broad wavelength tunability owing to the quantum size effect $[1,2]$. To date, the synthesis of nanocrystals with several shapes (spherical quantum dot [QD] [3], nanorod [4], nanowire [5], and nanodisk [6]) has been demonstrated with their respective unique properties. The shape of the nanocrystal defines the confinement of the charge carrier [7]. For example, spherical QDs confine the excitons in all three dimensions, whereas the nanowire provides the confinement in two dimensions. Recently, nanoplatelets (NPLs), which are also called colloidal quantum wells, have been demonstrated to exhibit even narrower luminescence compared with spherical QDs [8]. Several reports have been published on the synthesis and characterization of CdSe-based NPLs [9-12]. However, very few works have been performed to study the incorporation of NPLs in the device applications $[13,14]$.
In this Letter, we demonstrate the incorporation of chemically synthesized CdSe NPLs in a color-converted InGaN/GaN light-emitting diode (LED) as an exciton donor. The color conversion efficiency of the device employing CdSe/ZnS QDs (acceptors) as color-converter components was found to increase by $23 \%$ owing to the exciton transfer from the donor CdSe NPLs through Förster resonance energy transfer (FRET) process. Time-resolved fluorescence spectroscopy and photoluminescence excitation (PLE) measurements further confirmed that FRET occurs between the donor and acceptor nanocrystals.

A three-neck flask was properly cleaned prior to the synthesis of five-monolayer CdSe NPLs. As a first step, 1-octadecene (ODE) and cadmium myristate were added to the flask. The amounts of the former and latter materials were $15 \mathrm{~mL}$ and $170 \mathrm{mg}$, respectively. The system was pumped down, and the temperature was raised to $250^{\circ} \mathrm{C}$ under $\mathrm{N}_{2} / \mathrm{O}_{2}$ ambient. Twelve $\mathrm{mg}$ of Se were mixed with $1 \mathrm{ml}$ of ODE in a separate flask and added to the three-neck flask. The CdSe NPLs were grown within $10 \mathrm{~min}$, after which the reaction was terminated with the addition of $0.5 \mathrm{~mL}$ of oleic acid (OA), and the system was cooled to room temperature. Successful purification was carried out, which extracted the NPLs from the solution containing a mixture of NPLs and by-products. Transmission electron microscope (TEM) JEOL JEM-2010 was used to characterize the NPLs and QDs. CdSe/ZnS QDs were synthesized according to the recipe reported by Bawendi and co-workers, with slight modifications [3]. Photoluminescence (PL) and PLE measurements of chemically synthesized nanocrystals were carried out using an RF-5301PC Shimadzu spectrafluorophotometer. Absorbance of nanocrystals was measured with a UV-1800 Shimadzu spectrophotometer. To study the incorporation of CdSe NPLs as an exciton donor in color-converted LEDs, InGaN/GaN LEDs were grown by metal-organic chemical vapor deposition (MOCVD) on top of the $c$-plane (0001) polar sapphire substrates. The process was initiated with the growth of a low-temperature $\left(550^{\circ} \mathrm{C}\right)$ 
nucleation layer and was followed by the growth of a thick undoped GaN film. Five pairs of quantum wells ( $\mathrm{InGaN}$ ) and quantum barriers $(\mathrm{GaN})$ were introduced between the electron-blocking $(\mathrm{AlGaN})$ and $\mathrm{n}$-doped $\mathrm{GaN}$ layers. Finally, the wafer was capped with $200 \mathrm{~nm}$ pGaN. The PL measurements revealed the emission peak location to be at $450 \mathrm{~nm}$ (not shown here). Contacts were formed following the mesa etching and the deposition of a Ni/Au current spreading layer. Two color-converted devices were fabricated to examine the critical role of CdSe NPLs as the exciton donor. The first hybrid device (Device A) consists of the InGaN/GaN LED coated with $5 \mathrm{nmol}$ of $\mathrm{CdSe} / \mathrm{ZnS}$ QDs. The thickness of the colorconverter film was around $650 \mathrm{~nm}$. On the other hand, a mixture of CdSe/ZnS QDs and CdSe NPLs was applied onto the surface of the second device (Device B). The donor-acceptor ratio in Device B was 1:1. The closely packed nanocrystal films were integrated onto the fabricated LEDs to achieve full color conversion. Electroluminescence (EL) and optical power measurements were carried out using an integrating sphere connected to QE65000 model Ocean Optics spectrometer.

Figure 1 depicts the room temperature PL and absorbance curves of chemically synthesized CdSe NPLs. The NPLs were excited in solution, and the emission was collected by the detector. The incoming and detected beams were perpendicular to each other. NPLs, excited with $345 \mathrm{~nm}$ of excitation wavelength, exhibit extremely narrow luminescence with the bandwidth of $14 \mathrm{~nm}$. The PL peak is located at $545 \mathrm{~nm}$. The two peaks in the absorption spectrum are attributed to the electronlight hole $(512 \mathrm{~nm})$ and electron-heavy hole $(541 \mathrm{~nm})$ transitions. TEM and optical images of CdSe NPLs are shown in the insets of Fig. 1. The NPLs exhibit rectangle-like shapes with average dimensions of $10 \mathrm{~nm} \times 30 \mathrm{~nm}$. The optical image of in-solution luminescence of NPLs confirms the successful synthesis of green-emitting nanocrystals.

Figure 2(a) shows the PL and absorbance measurements of $\mathrm{CdSe} / \mathrm{ZnS}$ QDs which are used as color-converter components on the InGaN/GaN LEDs. The bandwidth of QDs is $40 \mathrm{~nm}$, and the emission is centered at $635 \mathrm{~nm}$. The absorbance curve confirms that the nanocrystals absorb better when the incoming photon has a shorter emission wavelength. The peaks in the absorption band are attributed to the electron-heavy hole and electron-light hole transitions corresponding to the CdSe core and $\mathrm{ZnS}$ shell. TEM and optical images of CdSe/ZnS QDs are

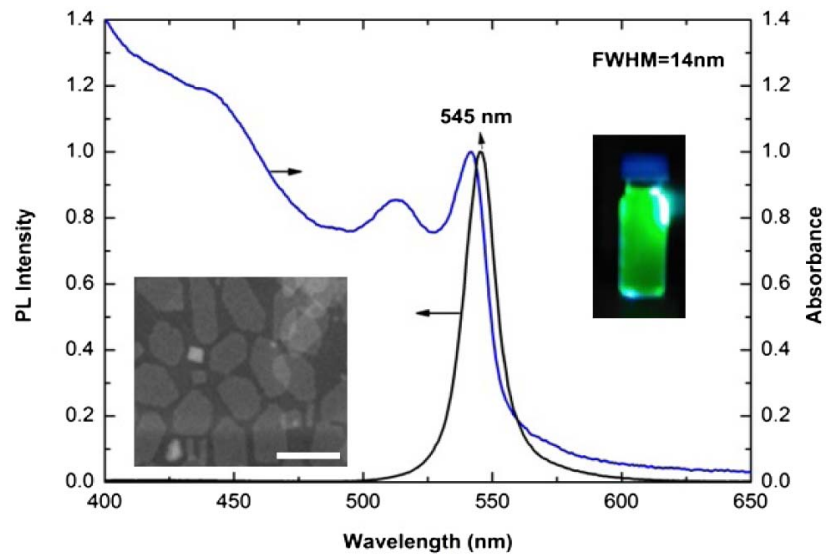

Fig. 1. PL and absorbance curves of CdSe NPLs. Insets: TEM and optical images of NPLs. The length of the scale bar is $30 \mathrm{~nm}$.
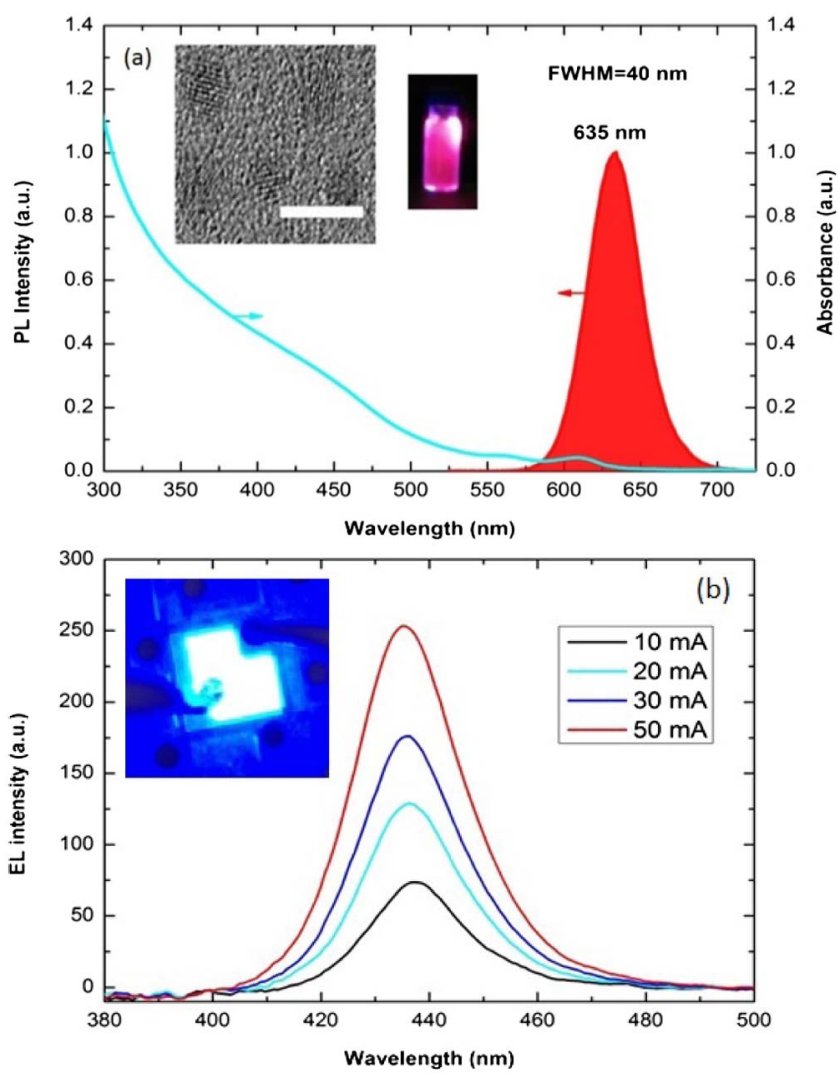

Fig. 2. (a) $\mathrm{PL}$ and absorbance curves of $\mathrm{CdSe} / \mathrm{ZnS}$ QDs. Insets: TEM image and optical images of CdSe/ZnS QDs. The length of the scale bar is $10 \mathrm{~nm}$. (b) EL spectra of InGaN/GaN LED at 10, 20, 30, and $50 \mathrm{~mA}$. Inset: optical image of InGaN/GaN LED in operation.

shown in the insets of Fig. 2(a). The average diameter of the nanocrystals is $6 \mathrm{~nm}$. Figure 2(b) presents the EL spectra of blue-emitting InGaN/GaN LED at 10, 20, 30, and $50 \mathrm{~mA}$. The EL peaks are centered at $438 \mathrm{~nm}$. The $12 \mathrm{~nm}$ blueshift compared with the PL measurement is attributed to the screening of the quantum confined stark effect (QCSE) with the injection of charge carriers. The optical image of conventional InGaN/GaN LED in operation is shown in the inset of Fig. 2(b).

The EL spectra of Device A and Device B at 10, 20, 30, and $50 \mathrm{~mA}$ current levels are presented in Fig. 3(a) with dashed and solid lines, respectively. The inset shows the schematic diagram of the upper layers of Device B utilizing both QDs (red dots) and NPLs (green plates). Both of the devices exhibit emission corresponding to the emission spectrum of color-converter $\mathrm{CdSe} / \mathrm{ZnS}$ QDs. A small redshift of emission compared with $\mathrm{PL}$ measurement of the CdSe/ZnS QDs in solution is ascribed to the change in the surrounding media. Moreover, a small size distribution of QDs $(<5 \%)$ results in nonradiative energy transfer (NRET) between them when these nanocrystals form close-packed solid films; the NRET process also contributes to the redshift with simultaneous quenching of smaller size QD intensity and the increase in the larger size QD intensity. The blue emission is fully absorbed with the introduction of colorconverter components. The intensity of Device $\mathrm{B}$ is higher than that of Device A at 10, 20,30, and $50 \mathrm{~mA}$ current levels. 

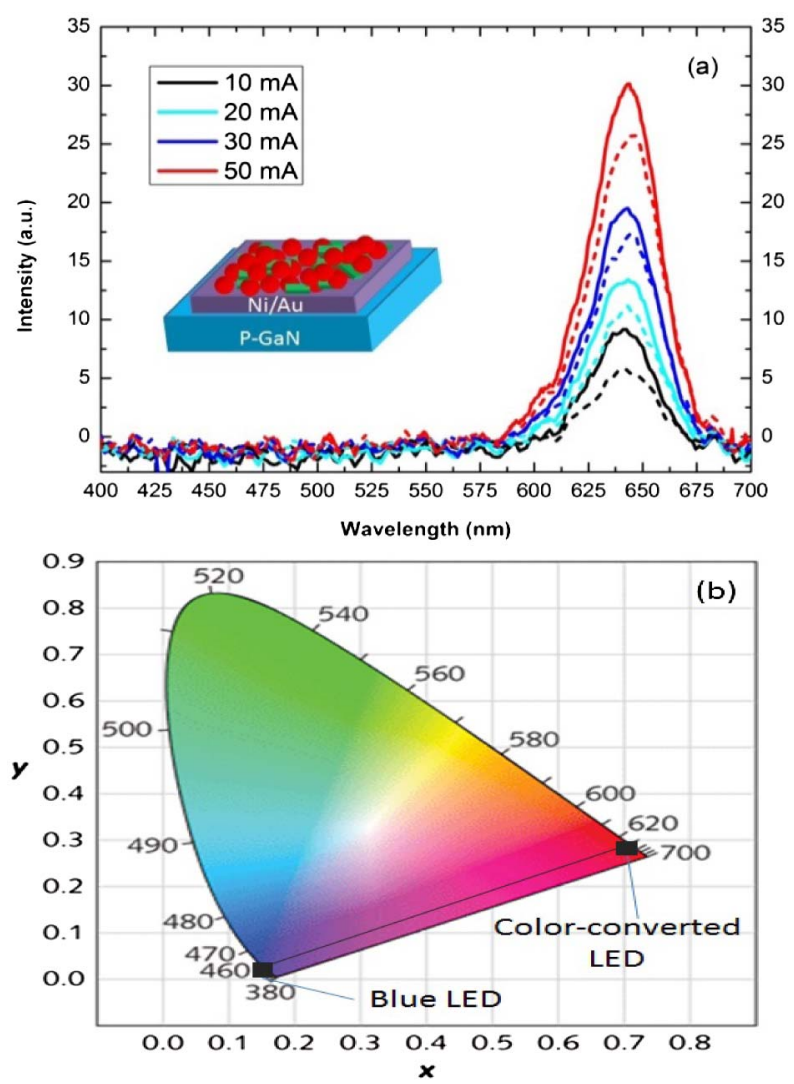

Fig. 3. (a) EL spectra of Device A (solid lines) and Device B (dashed lines) at 10, 20, 30, and $50 \mathrm{~mA}$. Inset: schematic diagram of Device B. (b) CIE chromaticity diagram showing the blue LED and the colorconverted LEDs.

Figure 3(b) depicts the chromaticity diagram with the blue and color-converted LEDs. The power conversion efficiency (the optical power ratios of the hybrid color-converted LED and the original blue emitting LED) of Device A and Device B are $14.35 \%$ and $17.64 \%$, respectively. The efficiency is increased by $23 \%$ with the incorporation of CdSe NPLs. Moreover, the heating-induced redshift in the EL spectrums is not observed in both devices owing to the operation of the devices at low injection current levels.

To reveal the mechanism behind the performance enhancement of the hybrid device utilizing CdSe NPLs, time-resolved photoluminescence spectroscopy analysis was carried out on the $\mathrm{CdSe} / \mathrm{ZnS} \mathrm{QD}$ solid films in the presence and in the absence of CdSe NPLs. The fluorescence lifetime measurements were carried out using a time correlated single-photon counting (SPC-150) system with an excitation laser at $375 \mathrm{~nm}$. The bandpass filter was used to record the decay rates of the samples at the acceptor QD emission. Since both measurements for the QD-only and QD-NPL samples were taken at the same temperature (room temperature), the thermal quenching effects can be neglected in the comparison of decay behaviors of these nanocrystals. Figure 4(a) shows the fluorescence decay curves of the CdSe/ZnS QDs in the above mentioned two conditions. The intensity of the QD solid film decays slower in the presence of the CdSe NPLs. The photon decay lifetimes is calculated from the decay curves by fitting them to bi-exponential functions. The amplitude-averaged decay lifetimes of the
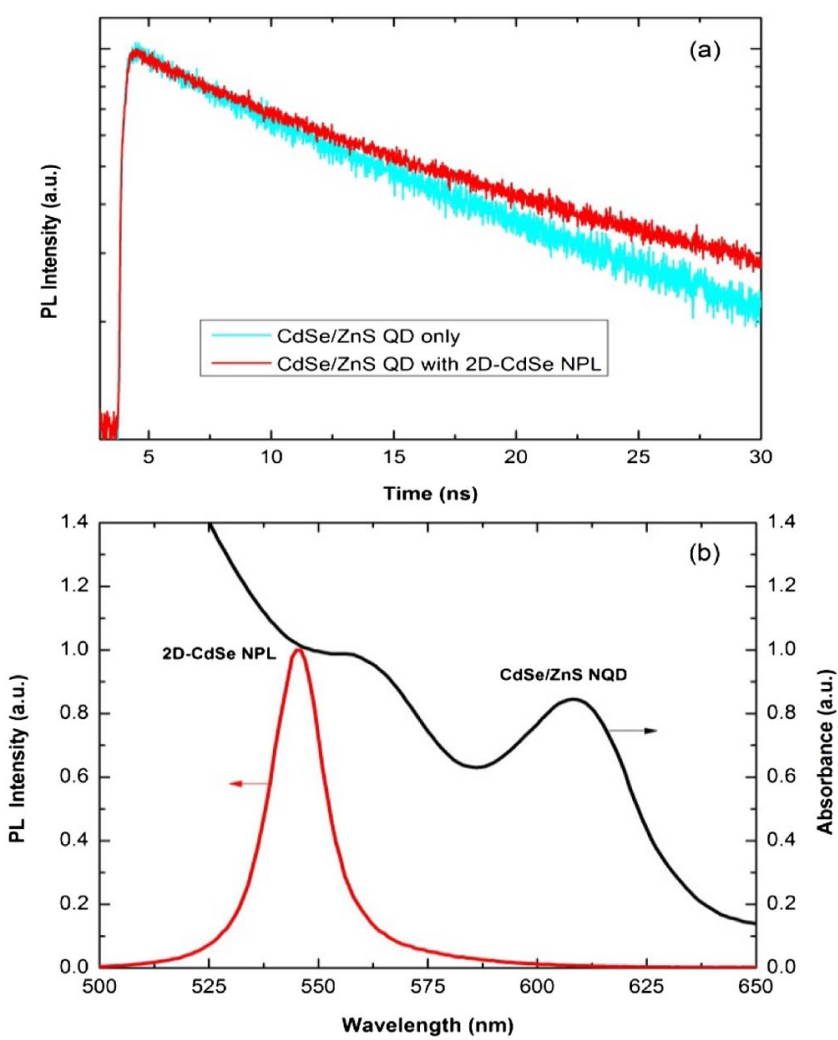

Fig. 4. (a) Time-resolved fluorescence decay traces of QD-only and QD-NPL solid films (b) PL intensity of CdSe NPLs and absorbance of $\mathrm{CdSe} / \mathrm{ZnS}$ QDs.

QD-only and the QD-NPL solid films are 12 and 15 ns, respectively. The $\chi^{2}$ parameters are close to 1 for the lifetime measurements on the QD-only and the QD-NPL films, respectively. The delayed decay of the QD film in the presence of CdSe NPLs is attributed to the FRET process occurring between the CdSe NPLs and CdSe/ZnS QDs. For an efficient FRET process to take place between these two types of nanocrystals, the absorbance of the acceptor nanocrystals (CdSe/ $\mathrm{ZnS}$ QDs) should have large overlap with the emission of the donor nanocrystals (CdSe NPLs). The emission of donor and the absorption of acceptor species used in this Letter are plotted in Fig. 4(b). As is clear from the figure, there is a significant overlap between the absorption and emission of acceptor and donor nanocrystals, respectively. This condition provides an efficient FRET process to take place between the donor-acceptor pairs. Moreover, by using the FRET model for the quantum well-quantum dot system with donor NPL lifetimes in the presence and in the absence of the acceptor QDs, we estimated the Förster radius (the donor-acceptor separation at which the FRET efficiency is 50\%) of this hybrid system to be $6.5 \mathrm{~nm}$. Such a large Förster radius value enables highly efficient exciton transfer in these donor-acceptor pairs, since our donors and acceptors are close-packed solid films with minimum separation; the separation between the NPLs and the center of QD are only limited by the diameter and the ligands of QDs.

To further confirm FRET from donor CdSe NPLs to acceptor $\mathrm{CdSe} / \mathrm{ZnS}$ QDs, we measured the PLE intensities of QD-only and QD-NPL solid films at several excitation 


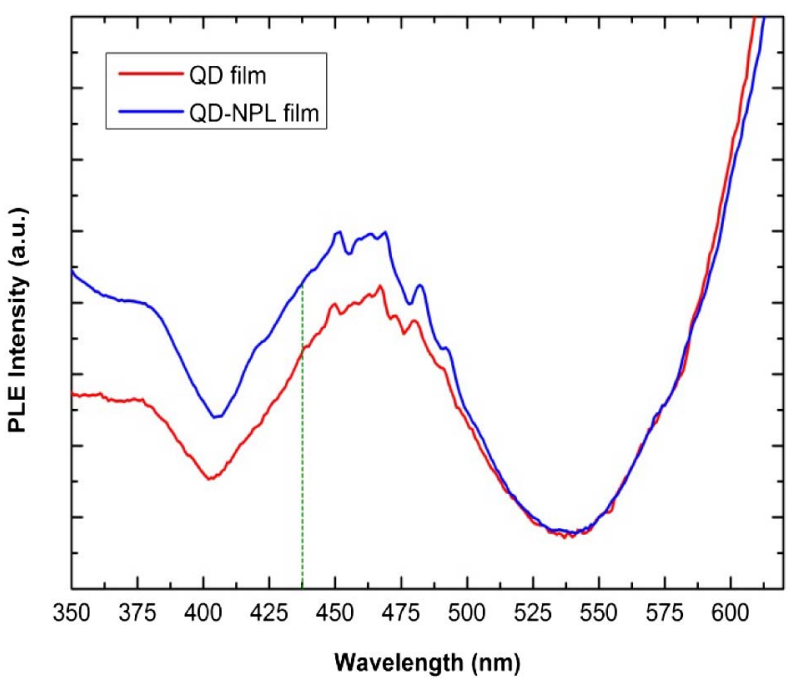

Fig. 5. PLE spectra of the QD-only and the QD-NPL solid films. The emission intensity was set to the peak emission wavelength of $\mathrm{CdSe} / \mathrm{ZnS}$ QDs in solid films.

energies. Figure 5 shows the PLE curves of these two samples which are excited with a source with excitation wavelength in the range of 350-620 nm; both measurements are set to monitor only the QD emission peak. When the QD-NPL film is excited with the source emitting at wavelength shorter than the absorption edge of NPLs, NPLs are excited simultaneously with QDs. At these excitation wavelengths, the intensity of QDs is significantly increased in hybrid NPL-QD film. This $\mathrm{PL}$ enhancement is attributed to the FRET process from NPLs to QDs. However, when the film is excited with an excitation wavelength longer than the absorption edge of the NPLs, only the QDs are optically pumped. At these high excitation wavelengths, intensity of QDs in the hybrid film is similar to that of the QD-only film. The PLE intensity of QD-NPL film is significantly increased when it is excited with $438 \mathrm{~nm}$ (dashed line in Fig. 5) which corresponds to the EL peak wavelength of the InGaN/GaN LED. This result suggests that optically excited NPLs strongly contribute to the emission enhancement of QDs through an efficient FRET process.

In summary, we demonstrated the application of chemically synthesized CdSe NPLs in the color-converted InGaN/GaN LED as an exciton source. The device fabricated with the incorporation of NPLs outperformed the LED without the NPLs in terms of EL intensity and optical power. The mechanism behind the enhancement was confirmed to be the efficient FRET process between the donor CdSe NPLs and the acceptor $\mathrm{CdSe} / \mathrm{ZnS}$ color-converter QDs. Time-resolved fluorescence decay experiments on the QD solid films revealed that there is a significant exciton migration between the CdSe NPLs and $\mathrm{CdSe} / \mathrm{ZnS}$ QDs which was confirmed with the increased decay lifetime of the acceptor QDs. PLE intensity measurements at the emission wavelength of the QDs further confirmed that NPLs markedly increase the emission intensity of the QDs when they are excited with the emission wavelength corresponding to the EL peak wavelength of the InGaN/GaN LEDs.

Funding. Singapore National Research Foundation (NRFCRP-11-2012-01, NRF-CRP-6-2010-2, NRF-RF-2009-09); Agency for Science, Technology and Research (A*STAR) (112 120 2009).

\section{REFERENCES}

1. A. M. Smith and S. Nie, Acc. Chem. Res. 43, 190 (2010).

2. V. Wood and V. Bulović, Nano Rev. 1, 5202 (2010).

3. B. O. Dabbousi, J. Rodriguez-Viejo, F. V. Mikulec, J. R. Heine, H. Mattoussi, R. Ober, K. F. Jensen, and M. G. Bawendi, J. Phys. Chem. B 101, 9463 (1997).

4. L. F. Xi and Y. M. Lam, J. Colloid Interface Sci. 316, 771 (2007).

5. N. P. Dasgupta, J. Sun, C. Liu, S. Brittman, S. C. Andrews, J. Lim, H. Gao, R. Yan, and P. Yang, Adv. Mater. 26, 2137 (2014).

6. V. F. Puntes, D. Zanchet, C. K. Erdonmez, and A. P. Alivisatos, J. Am. Chem. Soc. 124, 12874 (2002).

7. X. Peng, L. Manna, W. Yang, J. Wickham, E. Scher, A. Kadavanich, and A. P. Alivisatos, Nature 404, 59 (2000).

8. S. Ithurria, M. D. Tessier, B. Mahler, R. P. S. M. Lobo, B. Dubertret, and A. L. Efros, Nat. Mater. 10, 936 (2011).

9. M. Olutas, B. Guzelturk, Y. Kelestemur, A. Yeltik, S. Delikanli, and H. V. Demir, ACS Nano 9, 5041 (2015).

10. M. D. Tessier, C. Javaux, I. Maksimovic, V. Loriette, and B. Dubertret, ACS Nano 6, 6751 (2012).

11. M. D. Tessier, P. Spinicelli, D. Dupont, G. Patriarche, S. Ithurria, and B. Dubertret, Nano Lett. 14, 207 (2014).

12. B. Guzelturk, M. Olutas, S. Delikanli, Y. Kelestemur, O. Erdem, and H. V. Demir, Nanoscale 7, 2545 (2015).

13. A. G. Vitukhnovsky, V. S. Lebedev, A. S. Selyukov, A. A. Vashchenko, R. B. Vasiliev, and M. S. Sokolikova, Chem. Phys. Lett. 619, 185 (2015).

14. Z. Chen, B. Nadal, B. Mahler, H. Aubin, and B. Dubertret, Adv. Funct. Mater. 24, 295 (2014). 